Document downloaded from:

http://hdl.handle.net/10251/64660

This paper must be cited as:

Peidro Payá, D.; Díaz-Madroñero Boluda, FM.; Mula Bru, J.; Navalón Davó, A. (2015). A tabu search approach for production and sustainable routing planning decisions for inbound logistics in an automotive supply Chain. Enhancing Synergies in a Collaborative Environment. Part II:61-68. doi:10.1007/978-3-319-14078-0_7.

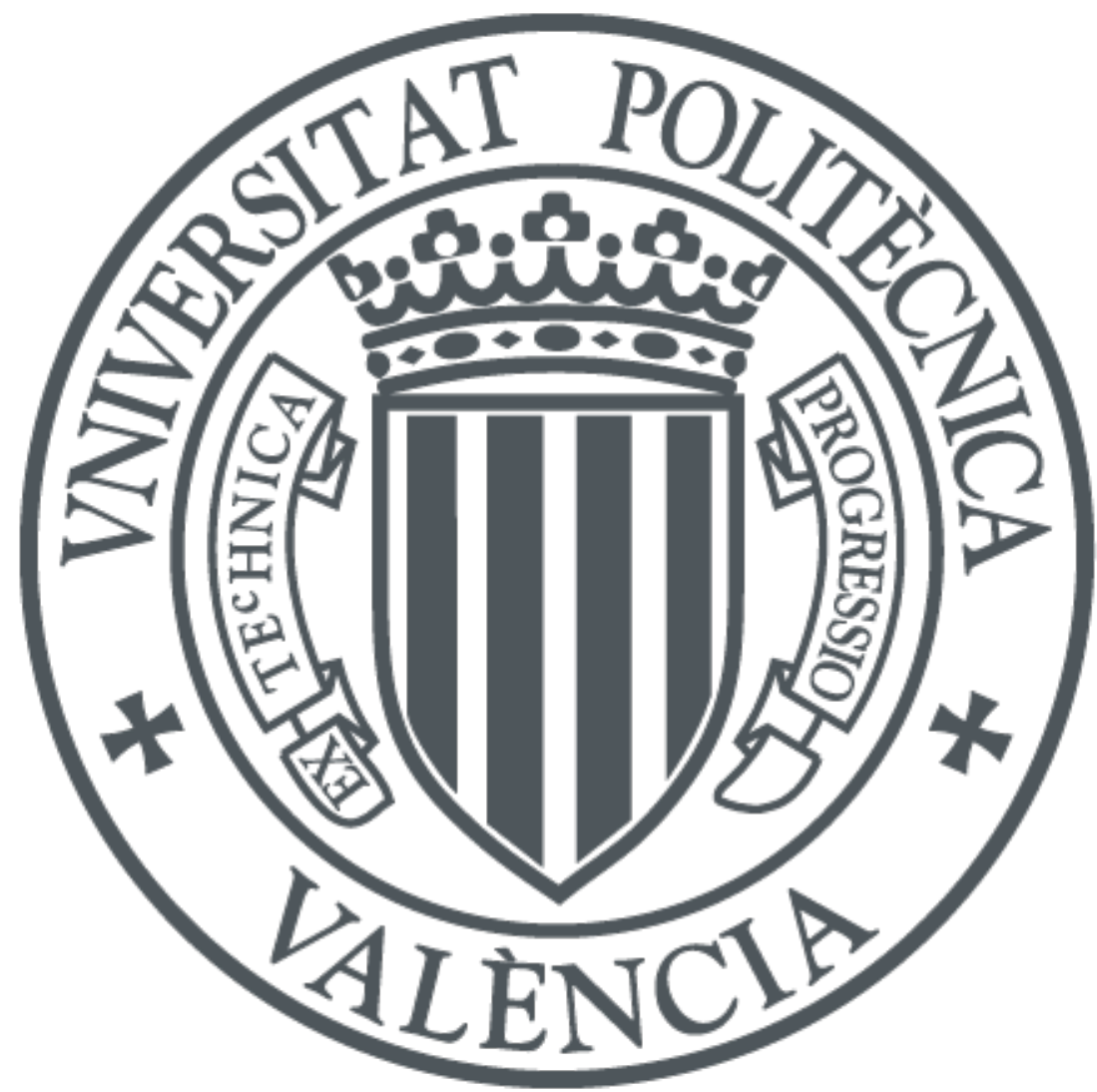

The final publication is available at

http://dx.doi.org/10.1007/978-3-319-14078-0_7

Copyright Springer International Publishing

Additional Information

The final publication is available at Springer via http://dx.doi.org/10.1007/978-3-319-140780_7 


\title{
A tabu search approach for production and sus- tainable routing planning decisions for inbound logistics in an automotive supply chain
}

\author{
Peidro $\mathrm{D}^{1}$, Díaz-Madroñero $\mathrm{M}^{1}$, Mula $\mathrm{J}^{1}$, Navalón $\mathrm{A}^{1}$
}

\begin{abstract}
In this paper, a mixed-integer mathematical programming model is proposed to address a production and routing problem related to inbound logistics processes in supply chains environments. This model is also enriched with sustainable issues related to routing decisions by introducing additional fuel consumption and pollutants emissions calculations into the objective function. For the solution methodology, a two-phase decoupled solution procedure based on exact algorithms for the production model and a tabu search algorithm for the routing model is adopted. Results of computational experiments performed with a realworld automotive supply chain confirm the efficiency of the proposed solution method in terms of total cost, fuel consumptions and CPU time.
\end{abstract}

Keywords: Material requirements planning; Vehicle routing; Green logistics; Tabu search

\section{Introduction}

In recent years, with the growing globalization of supply chains in high competitive business scenarios, industrial firms can not consider production planning and materials procurement as independent processes from transportation planning because their possible suboptimality from an economic perspective or their infeasibility due to capacity constraints. Moreover, apart from the economic cost viewpoint, these plans would include ecological and social issues, in order to meet needs of firms' stakeholders, and hence, improve their position with respect to their competitors. In this context, considering environmental criteria in the transport processes could have a tangible impact on responsible supply chain

${ }^{1}$ David Peidro, Manuel Díaz-Madroñero, Josefa Mula, Abraham Navalón (凹e-mail: fcodiama@cigip.upv.es)

Research Centre on Production Management and Engineering (CIGIP), Universitat Politècnica de València, Escuela Politécnica Superior de Alcoy, Plaza Ferrándiz y Carbonell, Alcoy, Spain 
management because a better use of natural resources and a reduction of greenhouse gases can be achieved.

The literature in production and routing problems (PRP), which integrate a lotsizing problem to determine production amounts and a vehicle routing problem (VRP) to determine delivery routes to distribute final products to customers, is scarce because the PRP is a recent research area focused on efficient solution procedures. In this sense, metaheuristics such as tabu search (TS) are considered a proper solution method for obtaining good quality solutions in reduced computational times (Bard \& Nananukul 2009; Armentano et al. 2011). However, all these proposed solution methods are evaluated by performing computational experiments with randomly generated instances and only evaluate plans from only an economic cost viewpoint, neglecting an ecological and social perspective. However, different green logistics practices and calculation methods have been incorporated recently into transport planning decisions in order to reducing fuel consumption (Jabali et al. 2012; Ubeda et al. 2011). Readers are referred to Demir et al. (2014) for a recent review of factors affecting fuel consumption in VRP optimization models.

After a review process, we highlight the following issues related to the PRP problem: (1) there is a need for PRP focused on inbound logistics processes; (2) PRP are validated, generally, with artificially generated instances, and hence, realworld applications are scarce; (3) new constraints and/or objective functions components can enrich current PRP models to tackle environmental requirements. In this sense, factors such as vehicle speed, transported load and gradient road factor can be considered significant. In this paper, we propose a mathematical programming planning for production and vehicle routing planning related to the procurement of parts and raw materials taking into account environmental issues such as fuel consumption and pollutants emissions, which issolved by a sequential approach based on a TS procedure. The paper is arranged as follows. Section 2 describes the fuel consumption calculation methodology considered in this study. Section 3 presents the problem and the corresponding formulation. Section 4 describes the solution methodology. Next, Section 5 evaluates the behavior of the proposed model in a real-world automobile firm. Finally, Section 6 provides conclusions and directions for further research.

\section{Fuel consumption calculation method}

In this study, MEET methodology has been adopted as a fuel consumption and pollutants calculation method and included in the proposed PRP mathematical programming model. Based on real-life experiments and diverse on-road measurements, Hickman et al. (1999) proposed a method for energy consumption calculations by using a "carbon-balance" based expression for pollutants such as $\mathrm{CO}_{2}, \mathrm{CO}$, unburned hydrocarbons and particulate matter emissions. The total 
emissions of each exhaust pollutant (i) correspond to the cold and hot emissions of each one, and can be calculated according to equations (1) to (4). Cold emissions $\left(E_{i, c o l d}\right)$ correspond to gases generated during each engine cold start, and can be defined according to equation (2), where $\varepsilon_{i, \text { cold }}$ is the cold emission factor for pollutant $i$ (in g/cold start) and $N$ is the number of cold starts in each route. Hot emissions are proportional to distance travelled in each vehicle route $d_{\text {route }}$, and depend on the hot emission factor $\varepsilon_{i, \text { hot }}$ (in $\mathrm{g} / \mathrm{km}$ ) which can be calculated by using equation (4) as a function of the average vehicle speed $v$. However, emissions factors of MEET refer to standard conditions (e.g. flat road gradient, empty vehicle, etc.) have to be corrected in order to take into account road gradient and load effects. The road gradient and load correction factors shown in equations (5) and (6), respectively, are used to take into account the effect of road slope and payload. Parameters $k_{1}, a, b, c, d, e, f, A_{6}, A_{5}, A_{4}, A_{3}, A_{2}, A_{1}, A_{0}, k_{2}, r, s, t, u$ and $\varepsilon_{i, \text { cold }}$ can be extracted from Hickman et al. (1999) for each type of road and vehicle, and $\mathrm{z}$ is the percentage of total load in each vehicle. Finally, the total value of hot emissions is given in equation (7).

$$
\begin{aligned}
& E_{i}=\sum_{\text {vehicle route }}\left(E_{i, \text { cold }}+E_{i, \text { hot }}\right) \\
& E_{i, \text { cold }}=\varepsilon_{i, \text { cold }} \cdot N \\
& E_{i, \text { hot }}=\varepsilon_{i, \text { hot }} \cdot d_{\text {route }} \\
& \varepsilon_{i, \text { hot }}=k_{1}+a v+b v^{2}+c v^{3}+\frac{d}{v}+\frac{e}{v^{2}}+\frac{f}{v^{3}} \\
& G C(v)=A_{6} v^{6}+A_{5} v^{5}+A_{4} v^{4}+A_{3} v^{3}+A_{2} v^{2}+A_{1} v+A_{0} \\
& L C(v)=\left(k_{2}+r v+s v^{2}+t v^{3}+\frac{u}{v}-1\right) z+1 \\
& E_{i, \text { hot }}=\varepsilon_{i, \text { hot }} \cdot G C(v) \cdot L C(v) \cdot d_{\text {route }}
\end{aligned}
$$

\section{Problem description and model formulation}

The PRP addressed in this paper can be defined in a network $G=(N, A)$, where $N$ represents the set of nodes comprising production plant and suppliers, and $A$ represents the set of arcs connecting the nodes, where $A=\{(s, v): s, v \in N, s \neq v\}$. Nodes are indexed by $s \epsilon\{0, \ldots, n\}$, where node 0 corresponds to the production plant, while suppliers are represented by $s \epsilon\{1, \ldots, n\}$ or the set $N s=N \backslash\{0\}$. In a finite planning horizon, composed of a set of equal planning periods $t=\{1, \ldots$, $T\}$, the production plant manufactures, in a set of productive resources $r$ with a production capacity $C A P_{r}$, a set of items represented by $i$. Moreover, extra production time $\left(T e x_{r t}\right)$ and iddle time $\left(T o c_{r t}\right)$ are also included. Manufactured 
products $p$, are considered finished goods composed of parts and raw materials according to the corresponding BOM. Finished goods and parts and raw materials can be stored in dedicated warehouses with capacity $C A P I_{i}$. Transportation of raw materials and parts from suppliers is done by a set of identical vehicles $K=\{1, \ldots$, $k\}$ with capacity $C A P T$ over the set of arcs $A$, which have an associated cost $c_{s v}$ to travel from node $s$ to node $v$. Each supplier can only be visited once per period. Transport routes are completed during one period and can start at any supplier and always finish at production plant. Moreover, travel times between nodes, waiting, loading and unloading times are not considered. Moreover, due to legal and labour restriccions transport routes can not exceed a maximum distance $M D$. The nomenclature defines the sets of indices, parameters and decision variables (Table $1)$.

Table 1 Nomenclature

\begin{tabular}{|c|c|c|c|}
\hline \multicolumn{4}{|l|}{ Sets } \\
\hline$T$ & set of time periods & $S, V$ & set of suppliers \\
\hline$I$ & set of products & $R$ & set of resources \\
\hline$J$ & set of parent products in BOM & $K$ & set of vehicles \\
\hline \multicolumn{4}{|c|}{ Parameters } \\
\hline$d_{i t}$ & demand of $i$ in $t$ & $c i_{i}$ & Unitary holding cost of $i$ \\
\hline $\operatorname{Inv} v_{i 0}$ & Initial inventory of $i$ & $\operatorname{crd}_{i}$ & Unitary backorder cost of $i$ \\
\hline$R d_{i 0}$ & Initial backorders of $i$ & ctoc $_{r}$ & Idle time cost in $r$ \\
\hline$R P_{i t}$ & Programmed receptions of $i$ in $t$ & ctex $_{r}$ & Overtime cost in $r$ \\
\hline$A_{i j}$ & Required quantity of $i$ to produce a unit & $\operatorname{cdis}_{S V}$ & Travel cost between $s$ and $v$ \\
\hline & of $j$ & $\operatorname{dis}_{s v}$ & Travel distance between $s$ and $v$ \\
\hline$a r_{i}$ & Unitary processing time of $i$ & $c p_{i}$ & $\begin{array}{l}\text { Unitary production or purchas- } \\
\text { ing cost of } i\end{array}$ \\
\hline$C A P_{r}$ & Production capacity of $r$ & CAPT & Vehicle capacity \\
\hline$C A P I_{i}$ & Warehouse capacity for $i$ & $M D$ & Maximum distance per route \\
\hline \multicolumn{4}{|c|}{ Decision variables } \\
\hline$P_{i t}$ & Production amount of $i$ in $t$ & Inv $v_{i t}$ & Inventory of $i$ int $t$ \\
\hline$Q_{i t k s}$ & $\begin{array}{l}\text { Purchasing amount of } i \text { in } t \text { transported } \\
\text { in } k \text { from } s\end{array}$ & $R d_{i t}$ & Backorders of $i$ in $t$ \\
\hline Toc $r t$ & Idle time in $r$ in $t$ & Tex $r t$ & Overtime in $r$ in $t$ \\
\hline$Y_{k s v t}$ & 1 if $k$ travels from $s$ to $v$ in $t$ & $X_{k s t}$ & 1 if $k$ visites $s$ in $t$ \\
\hline
\end{tabular}

Objective function

Min Z $=\sum_{i} \sum_{t} c p_{i} \cdot P_{i t}+\sum_{i} \sum_{t} \sum_{k} \sum_{s} c p_{i} \cdot Q_{i t k s}+\sum_{i} \sum_{t} c i_{i} \cdot I n v_{i t}+\sum_{i} \sum_{t} c r d_{i} \cdot R d_{i t}+$
$+\sum_{r} \sum_{t} c t o c_{r t} \cdot \operatorname{Toc}_{r t}+\sum_{r} \sum_{t} c t e x_{r t} \cdot T e x_{r t}+\sum_{t} \sum_{k} \sum_{s} \sum_{v} c d i s_{s v} \cdot Y_{k s v t}$

Subject to 


$$
\begin{array}{ll}
I N V T_{i, t-1}+P_{i t}+R P_{i t}+\sum_{k} \sum_{s} Q_{i k s s}-I N V T_{i t}-R d_{i, t-1}+R d_{i t}-\sum_{j=1}^{I} a_{i j} P_{j t}=d_{i t} \forall i \forall t \\
\sum_{i=1}^{I} A R_{i r} P_{i t}+T o c_{r t}-T e x_{r t}=C A P_{r t} & \forall r, \forall t \\
I N V_{i t} \leq C A P I_{i} & \forall i, \forall t \\
\sum_{s} \sum_{v} d_{v} t_{s v} \cdot Y_{k s v t} \leq M D & \forall k, \forall t \\
\text { Routing constraints }_{P_{i t,} Q_{i t k s}, I n v_{i t}, R d_{i t} \geq 0 \text { and integer }} & \forall i, \forall k, \forall t \\
\operatorname{Toc}_{r t}, \operatorname{Tex}_{r t} \geq 0 & \forall r, \forall t \\
Y_{k s v t}, X_{k s t} \in\{0,1\} & \forall k, \forall s, \forall v, \forall t
\end{array}
$$

The objective function (8) corresponds to the minimization of total costs relating to production, inventories, backlogs, overtime and undertime production costs, and routing costs over the planning horizon. Constraints (9) represent the inventory flow balance at the plant. Constraint (10) establishes the available capacity for normal, overtime production and iddle time. Constraint (11) establishes the inventory limits of each product at manufacturing plant . Constraint (12) establishes the distance limitation for each route and constraint (13) deals with the typical constraints for capacitated VRP. Constraints (14-16) define the variable decision domains. In order to include environmental and sustainable issues in the previous PRP model, the objective function (8) is modified to take into account the minimization of fuel consumption according to the MEET methodology.

\section{Solution approach}

A two-phase approach based on, firstly, the elaboration of a production and a material requirement plan and, secondly, the generation of a vehicle routing plan. According to Boudia et al. (2008), this uncoupled heuristic is quite standard in the industry. Production and material requirement plans are optimally solved by using a standard MIP solver which returns for each period the amounts to manufacture for each finished good, the amounts of parts and raw materials to order to each supplier, inventory and backorder levels, as well as idle and overtime times. The resulting planning is imposed on the inbound vehicle routing planning phase and cannot be modified.

\subsection{Tabu search}


Due to the combinatorial nature of VRP problem and in order to solve it in a reasonable computational time, a TS procedure (Glover \& McMillan 1986) has been developed. The algorithm works as follows:

1. An initial feasible solution $s$ is achieved by using a modified version of the Clarke \& Wright (1964) algorithm to address asymmetric VRP problems according to Vigo (1996).

2. The TS algorithm tries to find the best neighbourhood solution from the current solution by moving the positions of the nodes between the routes. This neighbour solution will be the next one in the solution space. This solution procedure removes a node from its current route and inserts it at a minimum cost into another route, based on the following steps: (1) for each node, it is looked for the best solution of moving it to other route without exceeding the capacity constraint; and (2) after all solutions have been calculated, the next neighbour solution is the best obtained that is not tabu or achieves the aspiration condition. Moreover, in this algorithm when a node is inserted into a route the position chosen is the one that minimizes the distance on this route.

3. The best solution obtained s' that it is not tabu or complies with the aspiration criterion will be the next feasible solution $s=s^{\prime}$ and the algorithm TS is restarted. This process is repeated until the stop criterion is found.

The main feature of TS is the tabu list that implements a diversification strategy. When a new neighbour solution is obtained, the pair node-route that has been moved is added to the tabu list and is set it with a tabu duration $\theta$, called tabu memory. This means that in the next $\theta$ iterations it is forbidden to re-inserting that node into that route. The value of the tabu duration $\theta$ is the key parameter that allows the success operation of the algorithm.

\section{Application to an automotive sector manufacturer}

The proposed model and solution approach have been validated as a tool for decsion-making related to the production planning and green transport planning for procurement parts in an European automotive sector manufacturer. This firm manufactures three finished goods in a single production plant in which parts and raw materials are procured from 21 different suppliers. Their facilities are close to the production plant, so that the duration of transport routes is minor than one day with a maximum of $635 \mathrm{~km}$. per each one. Moreover, it is assumed that there will always be enough vehicles available for the procurement of parts and raw materials with a total capacity of 13.6 linear meters per truck, therefore an only single time period has been considered. For emissions and fuel consumption calculations, a maximum speed average of $70 \mathrm{~km} / \mathrm{h}$ has been considered. Gradient factors have 
been obtained as a difference between elevations of start and end nodes in each arc.

The production and vehicle routing problems were developed with the MPL modeling language and solved by the CPLEX 12.1.0 solver in an Intel Core i5, at $2.80 \mathrm{GHz}$, with $6 \mathrm{~GB}$ of RAM. The proposed TS procedure to solve the vehicle routing problem was implemented in Java. A preliminary analysis was conducted to fine-tune the TS attributes. Two instances, with 10 and 21 suppliers, respectively, without considering fuel consumption minimization, were solved by using MPL and CPLEX solver with its defaults settings and compared to those results obtained by the TS algorithm. Purchasing amounts of parts and raw materials obtained from production and material requirement planning model are considered in order to determine collecting routes from suppliers. The results of the experiments related to the VRP problem by using CPLEX solver and TS procedure are presented in Table 2 for instances with 10 and 21 suppliers. For the instance problem with 10 suppliers, the results obtained by CPLEX solver and TS algorithm are identical in terms of solution cost and fuel consumption and similar in terms of CPU time. However, the TS procedure outperforms the results obtained by CPLEX solver for the instance problem with 21 suppliers. In this case, the TS algorithm provides a lower fuel consumption level and a better solution cost than CPLEX solver in a significantly shorter CPU time. In this sense, these better results are obtained in 0.607 seconds in comparison to more than 3 hours needed by CPLEX. Moreover, a better solution cost implies a lower travelled distance and a lower fuel consumption, and hence a lower level of pollutants and greenhouse emissions.

Table 2 Vehicle routing planning model results comparison

\begin{tabular}{ccccccc}
\hline $\begin{array}{c}\text { Number of } \\
\text { suppliers }\end{array}$ & \multicolumn{3}{c}{ CPLEX } & \multicolumn{3}{c}{ Tabu search } \\
\cline { 2 - 7 } & $\begin{array}{c}\text { Solution } \\
\text { cost }(€)\end{array}$ & $\begin{array}{c}\text { Solution } \\
\text { CPU } \\
\text { time (s) }\end{array}$ & $\begin{array}{c}\text { Fuel con- } \\
\text { sumption }(\mathrm{l})\end{array}$ & $\begin{array}{c}\text { Solution } \\
\text { cost }(€)\end{array}$ & $\begin{array}{c}\text { Solution } \\
\text { CPU } \\
\text { time (s) }\end{array}$ & $\begin{array}{c}\text { Fuel con- } \\
\text { sumption (l) }\end{array}$ \\
\hline 10 & 1091.44 & 0.34 & 292.11 & 1091.44 & 0.240 & 292.11 \\
21 & 1890.42 & 10888 & 532.92 & 1869.40 & 0.607 & 528.80 \\
\hline
\end{tabular}

\section{Conclusions}

This paper has proposed a mathematical programming model for production and sustainable routing planning decisions for inbound logistics processes in supply chain environments. A two-phase solution methodology based on a production and material requirement plan solved by a MIP solver and a VRP with fuel consumption minimization solved by a TS procedure has been adopted. The advantages of this proposal are related to: (1) the modelling of a new PRP for inbound logistics processes which includes sustainability issues such as fuel 
consumption and pollutants emissions; (2) the development of a highly effective TS procedure in finding good-quality solutions in short CPU times. With respect to the limitations of this proposal, we have described them through further research proposals: (1) consideration of travel times between nodes, waiting, loading and unloading times; (2) extend the set of nodes to include those suppliers located more than a day away; (3) allow multiple visits per period at suppliers for collecting parts and raw materials; (4) more accurately determination of gradient factors, by considering intermediate nodes in each arc and their corresponding elevations; (5) to develop solution procedures to tackle the proposed PRP in an integrated way, by considering the synchronization of production and inbound routing plans.

\section{Acknowledgements}

This work has been funded by the Universitat Politècnica de València projects: 'Material Requirement Planning Fourth Generation (MRPIV)' (Ref. PAID-05-12) and 'Quantitative Models for the Design of Socially Responsible Supply Chains under Uncertainty Conditions. Application of Solution Strategies based on Hybrid Metaheuristics' (PAID-06-12)

\section{References}

Armentano, V.A., Shiguemoto, A.L. \& Løkketangen, A., 2011. Tabu search with path relinking for an integrated productiondistribution problem. Computers and Operations Research, 38(8), pp.1199-1209.

Bard, J.F. \& Nananukul, N., 2009. The integrated production-inventory-distribution-routing problem. Journal of Scheduling, 12(3), pp.257-280.

Boudia, M., Louly, M.A.O. \& Prins, C., 2008. Fast heuristics for a combined production planning and vehicle routing problem. Production Planning and Control, 19(2), pp.85-96.

Clarke, G. \& Wright, J.W., 1964. Scheduling of Vehicles from a Central Depot to a Number of Delivery Points. Operations Research, 12(4), pp.568-581.

Demir, E., Bektaş, T. \& Laporte, G., 2014. A review of recent research on green road freight transportation. European Journal of Operational Research.

Glover, F. \& McMillan, C., 1986. The general employee scheduling problem. An integration of MS and AI. Computers \& Operations Research, 13(5), pp.563-573.

Hickman, J. et al., 1999. Methodology for calculating transport emissions and energy consumption,

Jabali, O., Van Woensel, T. \& de Kok, A.G., 2012. Analysis of Travel Times and CO 2 Emissions in Time-Dependent Vehicle Routing. Production and Operations Management, 21(6), pp.1060 1074.

Ubeda, S., Arcelus, F.J. \& Faulin, J., 2011. Green logistics at Eroski: A case study. International Journal of Production Economics, 131(1), pp.44-51.

Vigo, D., 1996. A heuristic algorithm for the asymmetric capacitated vehicle routing problem. European Journal of Operational Research, 89, pp.108-126. 